\title{
Calibration of a catadioptric omnidirectional vision system with conic mirror
}

\author{
J. Marcato Junior ${ }^{a, *}$, A.M.G. Tommaselli ${ }^{\mathrm{b}}$, M.V.A. Moraes ${ }^{\mathrm{b}}$ \\ ${ }^{a}$ FAENG, UFMS - Universidade Federal de Mato Grosso do Sul, Campus Universitário, 79070-900 Campo Grande, Brazil \\ b Department of Cartography, UNESP - Univ Estadual Paulista, Rua Roberto Simonsen 305, 19060-900 Presidente Prudente, Brazil
}

\section{A R T I C L E I N F O}

\section{Article history:}

Received 20 June 2015

Received in revised form 23 September 2015

Accepted 20 October 2015

Available online 22 January 2016

\section{Keywords:}

Photogrammetry

Catadioptric

Camera calibration

Fisheye lens

\begin{abstract}
A B S T R A C T
Omnidirectional vision systems that enable $360^{\circ}$ imaging have been widely used in several research areas, including close-range photogrammetry, which allows the accurate 3D measurement of objects. To achieve accurate results in Photogrammetric applications, it is necessary to model and calibrate these systems. The major contribution of this paper relates to the rigorous geometric modeling and calibration of a catadioptric, omnidirectional vision system that is composed of a wide-angle lens camera and a conic mirror. The indirect orientation of the omnidirectional images can also be estimated using this rigorous mathematical model. When calibrating the system, which is composed of a wide-angle camera and a conic mirror, misalignment of the conical mirror axis with respect to the camera's optical axis is a critical problem that must be considered in mathematical models. The interior calibration technique developed in this paper encompasses the following steps: wide-angle camera calibration; conic mirror modeling; and estimation of the transformation parameters between the camera and conic mirror reference systems. The main advantage of the developed technique is that it does not require accurate physical alignment between the camera and conic mirror axis. The exterior orientation is based on the properties of the conic mirror reflection. Experiments were conducted with images collected from a calibration field, and the results verified that the catadioptric omnidirectional system allows for the generation of ground coordinates with high geometric quality, provided that rigorous photogrammetric processes are applied. (C) 2015 International Society for Photogrammetry and Remote Sensing, Inc. (ISPRS). Published by Elsevier
\end{abstract}

B.V. All rights reserved.

\section{Introduction}

Omnidirectional vision systems have been widely used in several research fields, including robot navigation, telepresence, close-range photogrammetry and virtual reality (Kang and Szeliski, 1997; Yagi, 1999; Spacek, 2005; Sturm et al., 2011). Omnidirectional images can be acquired using a fisheye lens; moving cameras or optical elements; catadioptric systems (Baker and Nayar, 1999); and multiple cameras with divergent view (Sturm et al., 2011). The all-reflective system, which camera objective is based on mirrors, also enable the imagery acquisition with wide field of view (Richter et al., 2013).

Catadioptric systems present the advantage of generating omnidirectional images by using a single camera in combination with a mirror; this arrangement eliminates the problems of camera synchronization and image stitching. Several types of mirrors are used

\footnotetext{
* Corresponding author. Tel.: +55 673345 7495; fax: +55 1832231534 .

E-mail addresses: jose.marcato@ufms.br (J. Marcato Junior), tomaseli@fct.unesp. br (A.M.G. Tommaselli), antunesdemoraes@gmail.com (M.V.A. Moraes).
}

for this purpose, including curved cross-sections (elliptic, hyperbolic and parabolic) mirrors; planar mirrors arranged in a pyramid; and conic mirrors. However, catadioptric systems have also been developed by combining several cameras and a pyramid of mirrors (Nalwa, 1996; Tan et al., 2004).

The main advantage of combining a perspective camera with a hyperbolic mirror and combining an orthogonal camera with a parabolic mirror is that the single viewpoint property is fulfilled. Catadioptric systems achieving this property are called central catadioptric systems (Baker and Nayar, 1999).

Several techniques for calibrating central catadioptric systems have been developed (Barreto and Araújo, 2002; Scaramuzza et al., 2006; Mei and Rives, 2007; Luber and Reulke, 2010; Puig et al., 2011; Puig et al., 2012). Some of these techniques can also be applied to the calibration of cameras with fisheye lenses. Schöenbein et al. (2014) developed an efficient and accurate technique to use quasi-central catadioptric systems, in which the calibration is conducted with a non-central camera model (Agrawal et al., 2011). Agrawal et al. (2011) proposed a generalized 
calibration model based on polynomials for non-central catadioptric systems, which uses quadratic mirrors.

In general, catadioptric systems with conic mirrors do not satisfy the single viewpoint property and thus cannot be classified as central systems. However, Spacek (2005) presented some advantages of using an omnidirectional catadioptric system that is composed of a camera and a conic mirror over other catadioptric systems. The conic mirror combines the benefits of the planar mirrors without radial distortion and radial loss of resolution and the advantages of the rotationally symmetric catadioptric sensor, requiring short exposure and providing isotropic imaging. The manufacturing cost is also an advantage of this mirror.

Catadioptric systems with conic mirrors present multiple viewpoints when the nodal point of the camera lens is farther from the cone apex. The locus of the effective viewpoint is a circle with radius $d \cdot \cos (2 \tau)$, where $d$ is the distance from the camera perspective center (PC) to the conic mirror apex and $2 \tau$ is the angle in the apex of the conic mirror (Baker and Nayar, 1999). The geometry of this catadioptric system is presented in Section 2.3.

To have a single viewpoint, Lin and Bajcsy (2001) developed a catadioptric system in which the conic mirror apex coincides with the camera PC by cutting off the cone upper part, at the cost, however, of reducing the amount of incoming light and the imaging area. Another alternative is to build a conic mirror with an opening angle of $90^{\circ}$ (Spacek, 2003).

In general, the mathematical models for this type of system consider the camera optical axis to be rigorously aligned with the conic mirror axis (Yagi et al., 1994; Joung and Cho, 1998; Lin and Bajcsy, 2001; Spacek, 2003; Burbridge and Spacek, 2006; López-Nicolás and Sagues, 2010). However, this perfect alignment is unfeasible to achieve in practice, which introduces an additional source of error.

Burbridge et al. (2008) developed mathematical models in which the alignment between the conic mirror and camera axis is not necessary. The authors validated mathematical models that project from the object space to the image space and vice versa by using simulated data that were generated using the POVRay software. To the best of the authors' knowledge, calibration methods for a catadioptric system composed of a camera and a conic mirror were not applied in the aforementioned paper.

Cauchois et al. (1999) developed a calibration method for the SYCLOP (Conic SYstem for LOcalization and Perception). The developed mathematical model considers the conic mirror apex, the camera perspective center, the point in the object space and the normal to the cone in the reflection point to all be in the same plane, which is a simplification of the reflection in the conic mirror surface, as Burbridge et al. (2008) pointed out.

The aim of this work is to present a novel technique for the calibration of a catadioptric omnidirectional vision system that is composed of a wide-angle lens camera and a conic mirror for mobile applications. The main advantage is that the misalignment between the camera optical axis and conical mirror axis is modeled. In Section 2, the methods applied to achieve the main goal of this work and the results are presented and discussed. In Section 3, the exterior orientation procedure and results are presented. Finally, Section 4 presents the conclusions derived from the Sections 2 and 3.

\section{Omnidirectional vision system calibration}

The following sections present a description of the omnidirectional system calibration (Fig. 1) and its technical steps: camera calibration (Section 2.1); conic mirror modeling (Section 2.2); and estimating the transformation parameters between the camera and the conic mirror reference systems (Section 2.3).

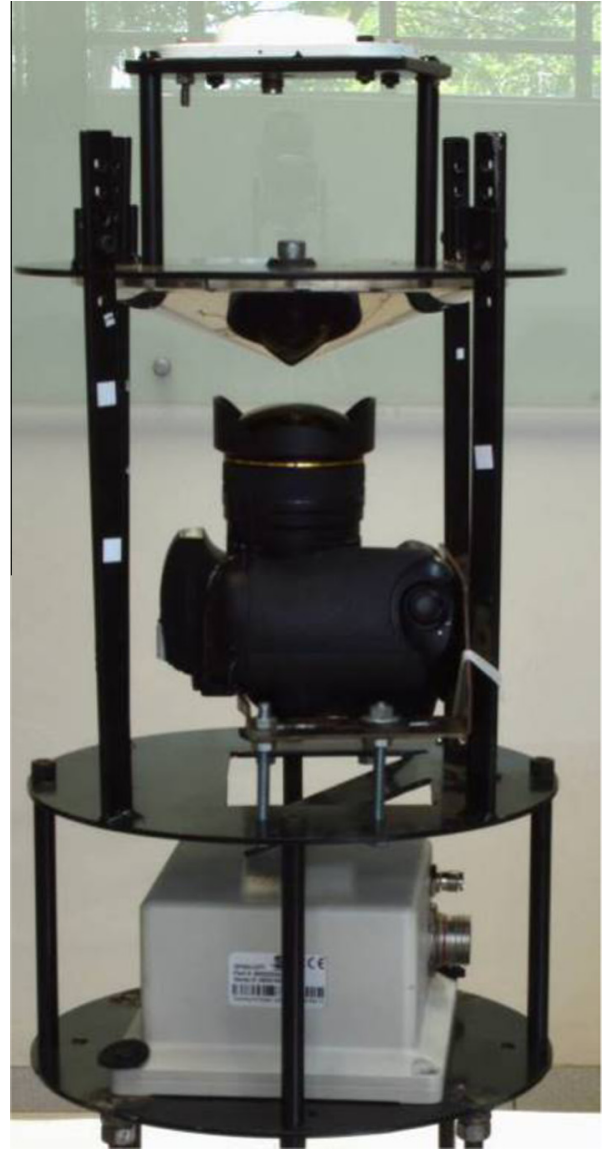

Fig. 1. Omnidirectional vision system composed of a wide-angle camera and a conic mirror. The system also has a double frequency SPAN-CPT/Novatel GNSS (Global Navigation Satellite System) receiver and an IMU (Inertial Measurement Unit) for direct image georeferencing.

\subsection{Wide-angle lens camera calibration}

A wide-angle lens was introduced in the proposed catadioptric system to increase both the coverage angle and the depth of field in a compact system with the camera positioned near $(4 \mathrm{~cm})$ to the mirror apex, enabling the acquisition of wider field of view when compared to a normal lens camera located at the same distance from the mirror.

In the experiments used to validate the proposed techniques, a Fuji Finepix S3 pro camera with a Bower-Samyang $8 \mathrm{~mm}$ lens was used. The Bower-Samyang lens that was used in this work is neither a fisheye lens nor a perspective lens. Charles (2009) classified this lens as quasi-stereographic because it is based on stereographic fisheye lens projection. In general, the perspective camera is calibrated using the collinearity mathematical model, which is based on perspective projection using points as features. Hughes et al. (2010) described the geometric properties of the models for a fisheye lens.

Abraham and Förstner (2005) and Schneider et al. (2009) presented mathematical models for calibrating a fisheye lens camera based on stereo-graphic, equi-distant, orthogonal and equi-solidangle projections. Schneider et al. (2009) combined these models with symmetric radial, decentering and affinity distortion models.

The calibration of the Fuji Finepix S3 pro camera with the quasifisheye Bower-Samyang $8 \mathrm{~mm}$ lens was performed using stereographic projection models (Eq. (1)). Tommaselli et al. (2014) and Marcato Junior et al. (2015) showed that the results obtained using the stereo-graphic, equi-distant, equi-solid-angle and orthogonal 
models are similar for this lens, and these models presented more accurate results in comparison to the perspective model, provided that these models were combined with the lens distortion models. In the current paper, only the stereo-graphic fisheye model was considered (Eq. (1)) because this is the model on which the Bower-Samyang lens is based.

$$
\begin{aligned}
& x^{\prime}=x_{0}+\Delta x+2 c \cdot \frac{X_{C}}{\sqrt{X_{C}^{2}+Y_{C}^{2}+Z_{C}^{2}}+Z_{C}} \\
& y^{\prime}=y_{0}+\Delta y+2 c \cdot \frac{Y_{C}}{\sqrt{X_{C}^{2}+Y_{C}^{2}+Z_{C}^{2}}+Z_{C}}
\end{aligned}
$$

where $X_{c}, Y_{c}$ and $Z_{c}$ are the 3D object point coordinates transformed to the camera reference system; $\Delta x$ and $\Delta y$ represent the systematic errors in the image coordinates - radial symmetric $\left(K_{1}, K_{2}\right.$ and $\left.K_{3}\right)$ and decentering distortions $\left(P_{1}\right.$ and $\left.P_{2}\right)$ (Conrady, 1919; Brown, 1971) and affinity $(A, B)$ (Habib and Morgan, 2003; El-Hakim, 1986); $c$ is the camera principal distance; $x_{0}$ and $y_{0}$ are the coordinates of the principal point; and $x^{\prime}$ and $y^{\prime}$ are the coordinates in the central image reference system parallel to the camera system with its origin at the image center.

The calibration of the Fuji Finepix S3 pro camera with the quasifisheye Bower-Samyang $8 \mathrm{~mm}$ lens was performed using the TMS (Triangulation with Multiple Sensors) software (Marcato Junior and Tommaselli, 2013), whose estimation process is based on the unified approach to least squares adjustment (Mikhail and Ackerman, 1976, p. 343). Twelve images from a 3D field calibration were used in the camera calibration. Tommaselli et al. (2014) and Silva et al. (2014) described the field calibration with ArUco coded targets (Garrido-Jurado et al., 2014) and the technique used for the automatic location of these coded targets in the images. Table 1 presents the estimated IOPs and their standard deviations.

The RMSE in $x^{\prime}$ and $y^{\prime}$ image coordinates were 0.58 and 0.62 pixels, respectively, for the stereo-graphic model. More details about the camera calibration, including the images residuals distribution, are presented in the work of Marcato Junior et al. (2015). It is important to mention that a reduction on the correlation between the interior and exterior orientation parameters occurs when considering the wide-angle lens, which is mainly due to the high scale variations caused by the wide field of view.

\subsection{Conic mirror modeling}

The conic mirror parameters can be estimated by using Eq. (2) with the least squares method from a suitable set of welldistributed sample points over the conic surface.

$R_{Z}+\sqrt{R_{X}^{2}+R_{Y}^{2}} \cdot \frac{h}{r}=R_{Z}+\sqrt{R_{X}^{2}+R_{Y}^{2}} \cdot \frac{1}{D}=0$,

where $h$ and $r$ are, respectively, the conic mirror height and radius and $R_{X}, R_{Y}$ and $R_{Z}$ are the 3D coordinates in the conic mirror reference system for the points that belong to the mirror surface. Some

Table 1

Estimated interior orientation parameters and their standard deviations for the stereo-graphic camera model (Marcato Junior et al., 2015).

\begin{tabular}{lll}
\hline & Estimated IOPs & Estimated standard deviation \\
\hline $\mathrm{c}(\mathrm{mm})$ & 8.4623 & 0.0011 \\
$\mathrm{x}_{0}(\mathrm{~mm})$ & -0.2545 & 0.0011 \\
$\mathrm{y}_{0}(\mathrm{~mm})$ & -0.3895 & 0.0010 \\
$\mathrm{~K}_{1}\left(\mathrm{~mm}^{-2}\right)$ & $-7.61 \times 10^{-4}$ & $5.32 \times 10^{-6}$ \\
$\mathrm{~K}_{2}\left(\mathrm{~mm}^{-4}\right)$ & $3.72 \times 10^{-7}$ & $8.49 \times 10^{-8}$ \\
$\mathrm{~K}_{3}\left(\mathrm{~mm}^{-6}\right)$ & $3.25 \times 10^{-9}$ & $4.16 \times 10^{-10}$ \\
$\mathrm{P}_{1}\left(\mathrm{~mm}^{-1}\right)$ & $-2.42 \times 10^{-5}$ & $1.67 \times 10^{-6}$ \\
$\mathrm{P}_{2}\left(\mathrm{~mm}^{-1}\right)$ & $9.67 \times 10^{-6}$ & $2.66 \times 10^{-6}$ \\
$\mathrm{~A}$ & $-1.48 \times 10^{-4}$ & $2.45 \times 10^{-5}$ \\
$\mathrm{~B}$ & $-8.25 \times 10^{-5}$ & $4.35 \times 10^{-5}$ \\
\hline
\end{tabular}

artificial points were painted over the conic mirror surface, and their coordinates were estimated through the bundle block adjustment of ten images from the mirror, which were acquired by a Nikon D3200 (24 Mpixels) digital camera with a Nikkor $30 \mathrm{~mm}$ lens. An example of an image used in this process is shown in Fig. 2. The mirror surface was positioned above a 2D flat calibration panel with ArUco coded targets, as presented in Fig. 2.

Nikon D3200 was calibrated previously by using the perspective model. In the bundle block adjustment with the TMS software, the coordinates of the painted marks over the mirror surface were estimated in the calibration panel reference system. However, the coordinates in Eq. (2) should be referenced to the conic mirror system. This system has its origin at the conic mirror apex, and the $X_{\text {cone }}$ and $Y_{\text {cone }}$ axes are parallel to the mirror base plane, as depicted in Fig. 2. The $Z_{\text {cone }}$ axis is defined considering a right-handed coordinate system. To relate the conic mirror to the panel reference system, a $3 \mathrm{D}$ rigid body transformation was used, as presented in Eq. (3).

$$
\left(\begin{array}{c}
X_{\text {cone }} \\
Y_{\text {cone }} \\
Z_{\text {cone }}
\end{array}\right)=\left(\begin{array}{l}
R_{X} \\
R_{Y} \\
R_{Z}
\end{array}\right)=R_{\text {Panel }}^{\text {Cone }}\left(\begin{array}{c}
X_{P}-X_{C} \\
Y_{P}-Y_{C} \\
Z_{P}-Z_{C}
\end{array}\right)
$$

The mathematical model used in the conic mirror surface fitting is attained by substituting Eq. (3) for Eq. (2). Six parameters were considered, including five parameters of the 3D rigid body transformation and the $D$ parameter, which is $r / h$. $R_{\text {Panel }}^{\text {Cone }}$ is the rotation matrix that relates the panel and the conic mirror reference systems. The angle kappa $(\kappa)$, rotation about $Z_{\text {cone }}$ axis, is constrained to zero to arbitrarily define the direction of the $X_{\text {cone }}$ and $Y_{\text {cone }}$ axes. $X_{C}, Y_{C}$ and $Z_{C}$ represent the conic mirror apex coordinates in the calibration panel reference system. $X_{P}, Y_{P}$ and $Z_{P}$ are the coordinates of a point in the calibration panel reference system; $X_{\text {cone }} Y_{\text {cone }}$ and $Z_{\text {cone }}$ are the coordinates of the same point in the conic mirror reference system. The parameters for the conic mirror modeling were estimated using the unified approach to least squares adjustment (Mikhail and Ackerman, 1976, p. 343), which was implemented in the Matlab software.

The coordinates of 25 points on the mirror surface, as estimated in the bundle adjustment, were used as observations in the conic mirror fitting, with a standard deviation of $0.1 \mathrm{~mm}$ for each component. This value was assumed based on the results achieved in the bundle block adjustment with ten images acquired using a Nikon D3200 digital camera. Table 2 presents the estimated parameters and their standard deviations.The chi-square test was applied, with a null hypothesis $H_{0}: \hat{\sigma}_{0}^{2}=\sigma_{0}^{2}$ and the alternative hypothesis $H_{1}: \hat{\sigma}_{0}^{2}>\sigma_{0}^{2}$. The null hypothesis was accepted with a significance level of 5\%. This result indicates that the residuals in the 25 coordinate points are consistent with the assumed standard deviation of $0.1 \mathrm{~mm}$. Fig. 3 shows graphically the residuals' distribution, and it is evident that the residuals present a random behavior and are higher in the $\mathrm{Z}$ component but do not exceed $0.15 \mathrm{~mm}$.

In the bundle adjustment with ten images, the coordinates of 21 points on the mirror base plane (Fig. 2b) in the calibration panel reference system were also estimated. These coordinates were transformed to the conic mirror reference system using the parameters of the rigid body transformation presented in Table 2 . The coordinates of these points on the mirror base plane and those referenced to the conic mirror coordinate system are crucial for estimating the transformation parameters between the conic mirror and camera reference systems (Section 2.3).

\subsection{Estimation of the transformation parameters between camera and conic mirror reference systems}

The current process is necessary because of the misalignment of the conic mirror axes in relation to the camera optical axes. Fig. 4 


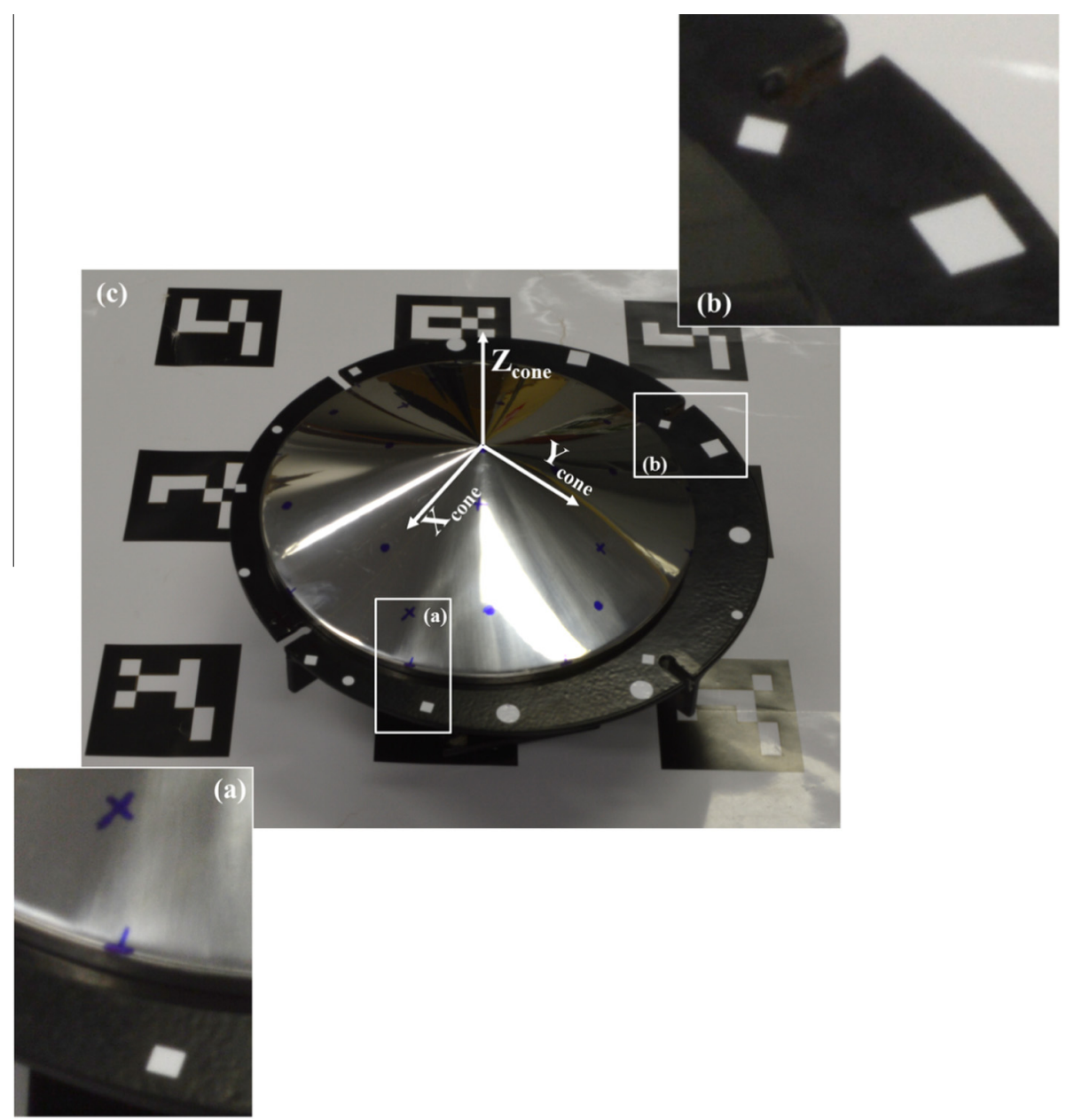

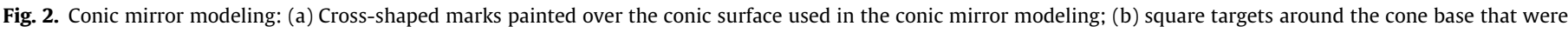

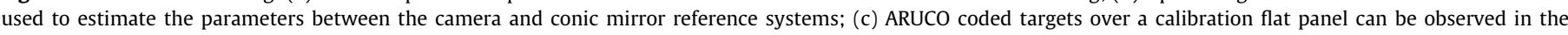
background.

Table 2

Parameters and standard deviation estimated in steps of conic mirror modeling.

\begin{tabular}{llllll}
\hline$\omega_{C}\left({ }^{\circ}\right)$ & $\phi_{C}\left({ }^{\circ}\right)$ & $X_{C}(\mathrm{~m})$ & $Y_{C}(\mathrm{~m})$ & $Z_{C}(\mathrm{~m})$ & $D$ \\
\hline 0.39960 & 0.06920 & 0.5844 & 0.4162 & 0.1434 & 2.6186 \\
$(0.06760)$ & $(0.06404)$ & $(0.0003)$ & $(0.0003)$ & $(0.0001)$ & $(0.0065)$ \\
\hline
\end{tabular}

Redundancy: 19 .

apriori variance factor $\left(\sigma_{0}\right): 1.0$.

aposteriori variance factor $\left(\widehat{\sigma_{0}}\right): 1.04$.

depicts the acquisition geometry for the developed omnidirectional vision system and emphasizes the three reference systems: camera, conic mirror and object space.

The coordinates of the 21 points on the mirror base plane that were transferred to the conic mirror system were used in the current step. An image collected with the omnidirectional vision system (Fig. 5) was used. The image coordinates of the 21 points on the mirror base were manually measured using the MID software (Tommaselli and Reiss, 2005). A standard deviation of 1 pixel was considered for the image observations because of the manual measurement.

The parameters (three orientation angles and three offsets) were estimated using the TMS software via a space resection with the stereo-graphic model. The points on the mirror base were considered control points, with a standard deviation of $0.1 \mathrm{~mm}$ for all coordinate components. Table 3 presents the estimated parameters.
The analysis of Table 3 shows that the aposteriori variance factor is smaller than the apriori variance factor. This result indicates that the observation residuals are smaller than the assumed standard deviations: 1 pixel to the image observations and $0.1 \mathrm{~mm}$ to the $3 \mathrm{D}$ coordinates in the conic mirror system.

The three steps of the system calibration (Sections 2.1, 2.2 and 2.3) were performed in sequential steps to cope with correlations between parameters.

\section{Exterior omnidirectional image orientation}

The first step to determine the exterior orientation is to estimate the reflection point coordinates in the conic mirror surface of the GCPs. These coordinates can be estimated using the inverse collinearity equations (Eq. (4)), with some minor adaptations that are detailed below.

$$
\begin{aligned}
& R_{X}=X_{P C}+\left(R_{Z}-Z_{P C}\right) \cdot \frac{r_{11} \cdot\left(x_{p}^{\prime}\right)+r_{21} \cdot\left(y_{p}^{\prime}\right)-r_{31} \cdot(c)}{r_{13} \cdot\left(x_{p}^{\prime}\right)+r_{23} \cdot\left(y_{p}^{\prime}\right)-r_{33} \cdot(c)} \\
& R_{Y}=Y_{P C}+\left(R_{Z}-Z_{P C}\right) \cdot \frac{r_{12} \cdot\left(x_{p}\right)+r_{22} \cdot\left(y_{p}^{\prime}\right)-r_{32} \cdot(c)}{r_{13} \cdot\left(x_{p}^{\prime}\right)+r_{23} \cdot\left(y_{p}^{\prime}\right)-r_{33} \cdot(c)}
\end{aligned}
$$

where $R_{X}, R_{Y}$ and $R_{Z}$ are the reflection point coordinates; $X_{P C}, Y_{P C}$ and $Z_{P C}$ are the coordinates of the camera perspective center in the conic mirror reference system; $r_{i, j}(1 \leqslant i, j \leqslant 3)$ are the rotation matrix

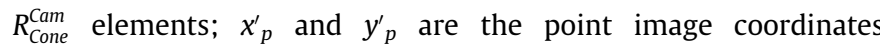



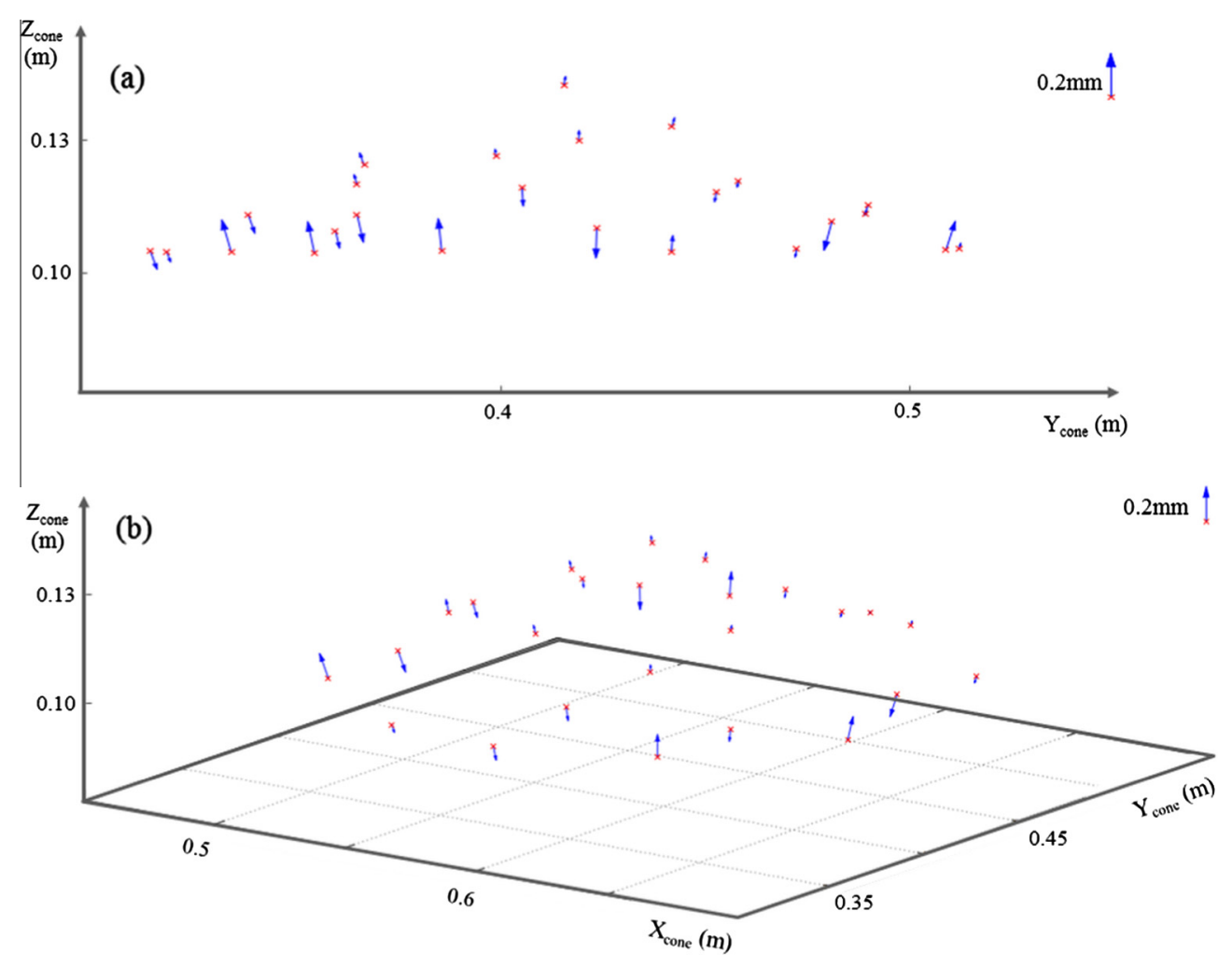

Fig. 3. Residual distribution after cone fitting: (a) side view; (b) perspective view.

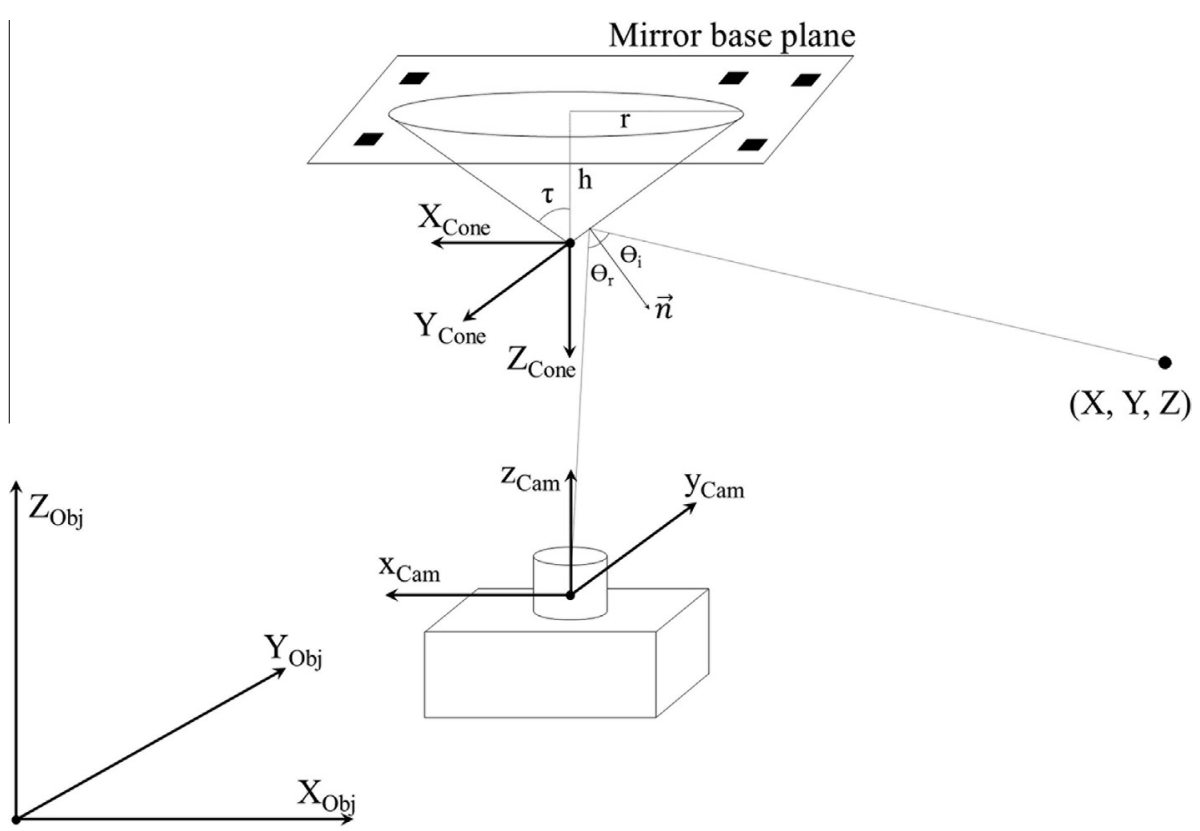

Fig. 4. Reference systems considered in the calibration of the developed omnidirectional vision system.

considering the perspective projection; and $c$ is the camera principal distance. In Eq. (4), there are three unknowns $\left(R_{X}, R_{Y}\right.$ and $\left.R_{Z}\right)$ and two equations. To estimate the coordinates of the reflection point, the conic mirror equation (Eq. (2)) is also considered. The coordinate $R_{Z}$ is calculated for each $R_{X}$ and $R_{Y}$ by using Eq. (2) with the estimated parameters.

To estimate the reflection point coordinates, photogrammetric monoplotting is used (Makarovik, 1973). In this iterative tech- nique, an initial value for $R_{Z}$ is first assumed and is usually an average value $(-0.020$, which is half of the conic mirror height); subsequently, the $R_{X}{ }^{(1)}$ and $R_{Y}{ }^{(1)}$ coordinates are calculated using Eq. (4). Then, the $R_{Z}^{(1)}$ coordinate is calculated again by using the $R_{X}{ }^{(1)}$ and $R_{Y}{ }^{(1)}$ values with Eq. (2). It is an iterative process, and the convergence criterion adopted was $\left|R_{Z}^{(i)}-R_{Z}^{(i-1)}\right|<0.1 \mu \mathrm{m}$.

Eq. (4) was developed for the perspective projection; however, the stereo-graphic projection was used in the camera calibration, 


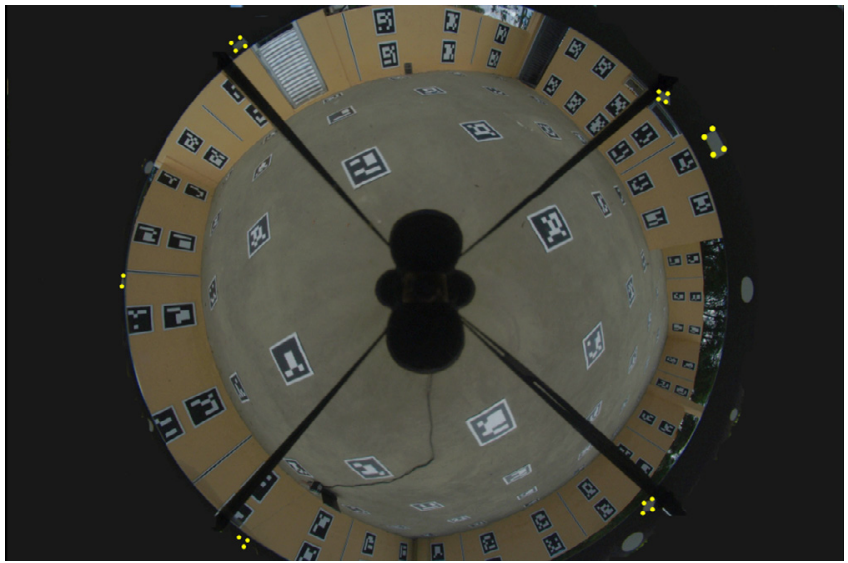

Fig. 5. Points (highlighted with yellow dots) on the mirror base used to estimate the parameters between the conic mirror and photogrammetry reference systems. The calibration field with coded targets is also shown. (For interpretation of the references to color in this figure legend, the reader is referred to the web version of this article.)

\section{Table 3}

Parameters and standard deviation relating the conic mirror and camera reference systems estimated with the TMS software through a space resection with the stereographic model.

\begin{tabular}{llllll}
\hline$\omega_{\text {Cone }}^{\text {Photo }}\left({ }^{\circ}\right)$ & $\phi_{\text {Cone }}^{\text {Photo }}\left({ }^{\circ}\right)$ & $\kappa_{\text {Cone }}^{\text {Photo }}\left({ }^{\circ}\right)$ & $X_{P C}(\mathrm{~m})$ & $Y_{P C}(\mathrm{~m})$ & $Z_{P C}(\mathrm{~m})$ \\
\hline 0.71758 & 180.90440 & 184.53864 & -0.0017 & 0.0023 & 0.0335 \\
$(0.02158)$ & $(0.01958)$ & $(0.010539)$ & $(0.0001)$ & $(0.0001)$ & $(0.0001)$ \\
\hline
\end{tabular}

apriori variance factor $\left(\sigma_{0}\right): 1.0$.

aposteriori variance factor $\left(\widehat{\sigma_{0}}\right): 0.74$.

and a correction is thus required. The image coordinates of the camera $\left(x^{\prime}, y^{\prime}\right)$ are transformed to the perspective projection coordinates $\left(x_{p}^{\prime}, y_{p}^{\prime}\right)$ by using the following steps:

(a) compute and apply corrections $(\Delta x, \Delta y)$ for the measured image coordinates $\left(x^{\prime}, y^{\prime}\right)$ (symmetric radial, decentering and affinity);

(b) compute the radial distance $r^{\prime}$ :

$$
r^{\prime}=\left(\left(x^{\prime}-x_{0}-\Delta x\right)^{2}+\left(y^{\prime}-y_{0}-\Delta y\right)^{2}\right)^{1 / 2}
$$

(c) compute $\alpha$ angle between the incoming ray and the $z_{\text {photo }}$ axis considering the stereo-graphic projection model (Schneider et al., 2009):

$$
\alpha=2 \cdot \operatorname{arctg}\left(r^{\prime} / 2 c\right)
$$

(d) compute the radial distance corresponding to the perspective projection $\left(r_{p}^{\prime}\right)$ and $\theta$ angle:

$$
\begin{aligned}
& r_{p}^{\prime}=c \cdot \operatorname{tg}(\alpha) \\
& \theta=\operatorname{arctg}\left(\left(y^{\prime}-y_{0}-\Delta y\right) /\left(x^{\prime}-x_{0}-\Delta x\right)\right)
\end{aligned}
$$

(e) finally, compute the $x_{p}^{\prime}$ and $y_{p}^{\prime}$ coordinates corresponding to the perspective projection using $r_{p}^{\prime}$ and $\theta$ angle.

A restriction to this process occurs when the $\alpha$ angle approaches $90^{\circ}$ because $r_{p}^{\prime}$ vanishes to infinity. Because the camera is not full frame, the maximum coverage angle does not reach $180^{\circ}$, and consequently, this restriction does not occur in practice. Fig. 6 depicts the elements involved in the aforementioned process. Then, the coordinates of the reflection point are estimated by using the monoplotting technique, described previously.

The parameters relating the conic mirror and object space reference systems can be estimated by using Eqs. (2), (10) and (12).

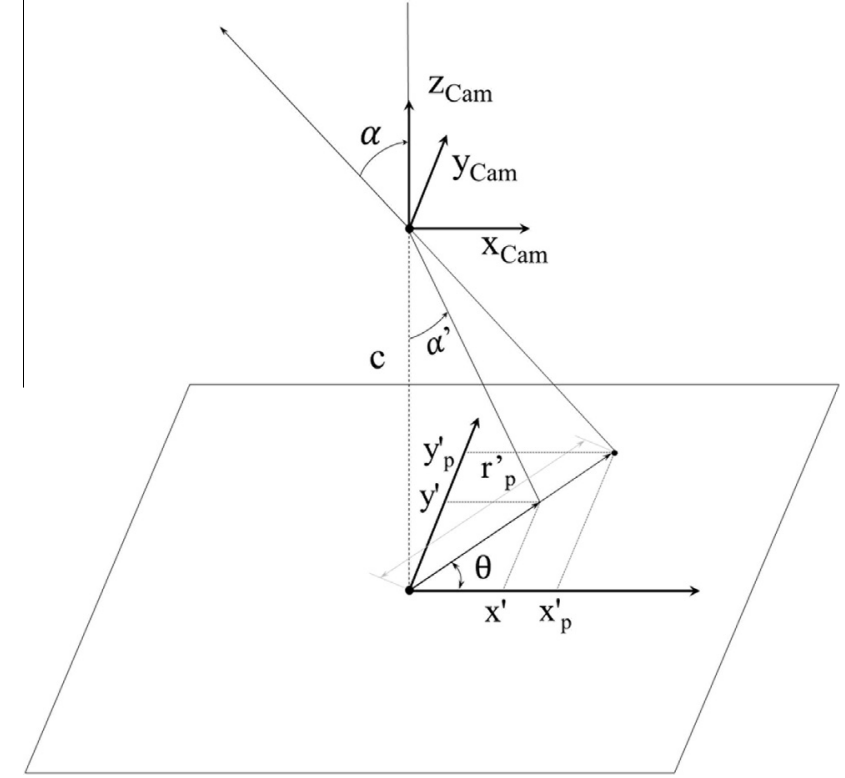

Fig. 6. Transformation applied to the image coordinates to use the inverse collinearity equations for the stereo-graphic fisheye projection.

These equations describe the reflection of a ray in a conic mirror (Burbridge et al., 2008):

(1) The reflection point coordinates must be on the conic mirror surface (Eq. (2)).

(2) The angle $\left(\theta_{i}\right)$ between the normal to the conic mirror $(\vec{n})$ at the reflection point $(R)$ and the vector from the object point $(P)$ to the reflection point must be equal to the angle $\left(\theta_{r}\right)$ between the normal and the vector from the camera PC $(C)$ to the reflection point:

$\frac{C-R}{\|C-R\|} \cdot \vec{n}-\frac{P-R}{\|P-R\|} \cdot \vec{n}=0$

The development of Eq. (9) generates the second equation used in the estimation of the exterior orientation parameters.

$$
\begin{aligned}
& \frac{\left(\left(X_{P C}-R_{X}\right) R_{Z} R_{X}+\left(Y_{P C}-R_{Y}\right) R_{Y} R_{Z}+\left(Z_{P C}-R_{Z}\right)\left(-R_{X}^{2}-R_{Y}^{2}\right)\right)^{2}}{\left(X_{P C}-R_{X}\right)^{2}+\left(Y_{P C}-R_{Y}\right)^{2}+\left(Z_{P C}-R_{Z}\right)^{2}} \\
& -\frac{\left(\left(X_{\text {cone }}-R_{X}\right) R_{Z} R_{X}+\left(Y_{\text {cone }}-R_{Y}\right) R_{Y} R_{Z}+\left(Z_{\text {cone }}-R_{Z}\right)\left(-R_{X}^{2}-R_{Y}^{2}\right)\right)^{2}}{\left(X_{\text {cone }}-R_{X}\right)^{2}+\left(Y_{\text {cone }}-R_{Y}\right)^{2}+\left(Z_{\text {cone }}-R_{Z}\right)^{2}}=0
\end{aligned}
$$

(3) The normal to the conic mirror at the reflection point must be in the same plane as the object point, the camera PC, and the reflection point:

$$
((P-C) \times(P-R)) \cdot \vec{n}=0
$$

Finally, the development of Eq. (11) provide the third equation:

$$
\begin{aligned}
& \left(\left(Y_{\text {cone }}-Y_{P C}\right)\left(Z_{\text {cone }}-R_{Z}\right)-\left(Z_{\text {cone }}-Z_{P C}\right)\left(Y_{\text {cone }}-R_{Y}\right)\right) R_{Z} R_{X} \\
& \quad+\left(\left(Z_{\text {cone }}-Z_{P C}\right)\left(X_{\text {cone }}-R_{X}\right)-\left(X_{\text {cone }}-X_{P C}\right)\left(Z_{\text {cone }}-R_{Z}\right)\right) R_{Z} R_{Y} \\
& +\left(\left(X_{\text {cone }}-X_{P C}\right)\left(Y_{\text {cone }}-R_{Y}\right)-\left(Y_{\text {cone }}-Y_{P C}\right)\left(X_{\text {cone }}-R_{X}\right)\right) \\
& \quad \times\left(-R_{X}^{2}-R_{Y}^{2}\right)=0
\end{aligned}
$$

The 3D point coordinates referenced to the conic mirror system $\left(X_{\text {cone }}, Y_{\text {cone }}, Z_{\text {cone }}\right)$ in Eqs. (10) and (12) are rewritten with the coordinates of the same point in the object space $(X, Y, Z)$ through a rigid body transformation (Eq. (13)). 


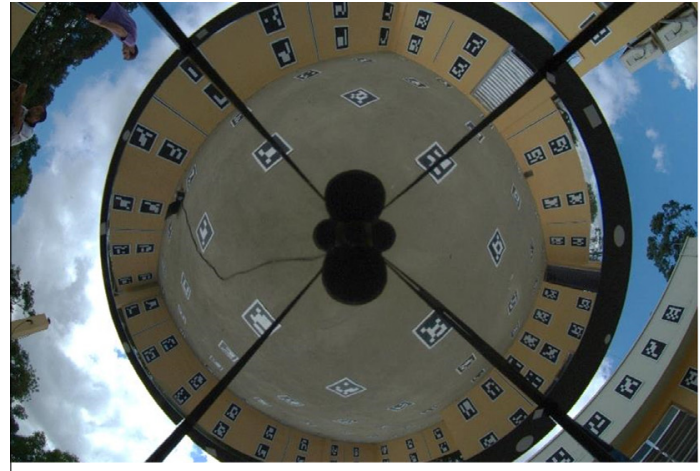

(a)

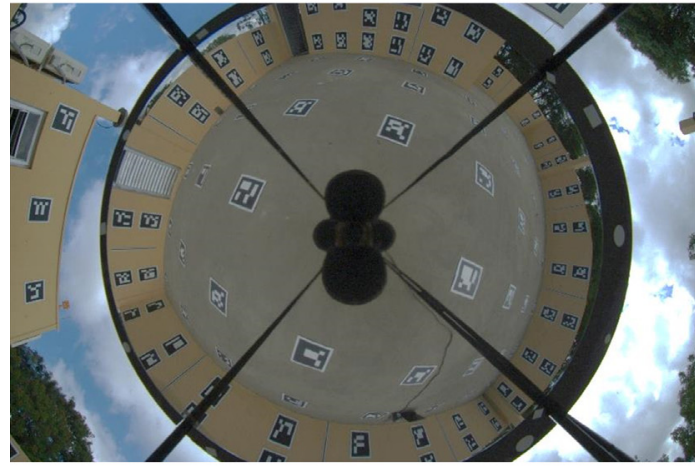

(c)

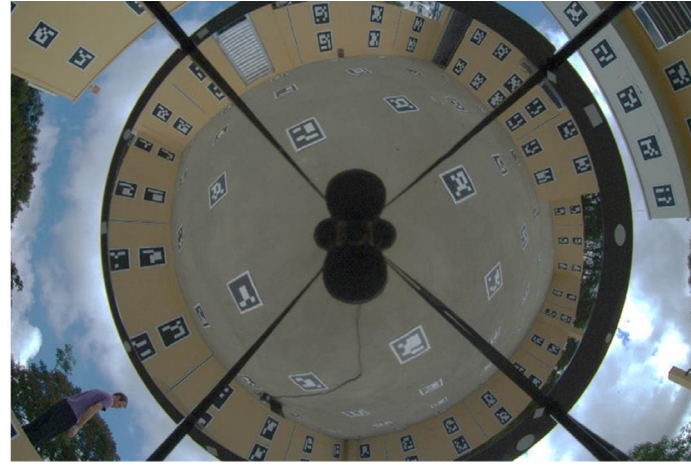

(b)

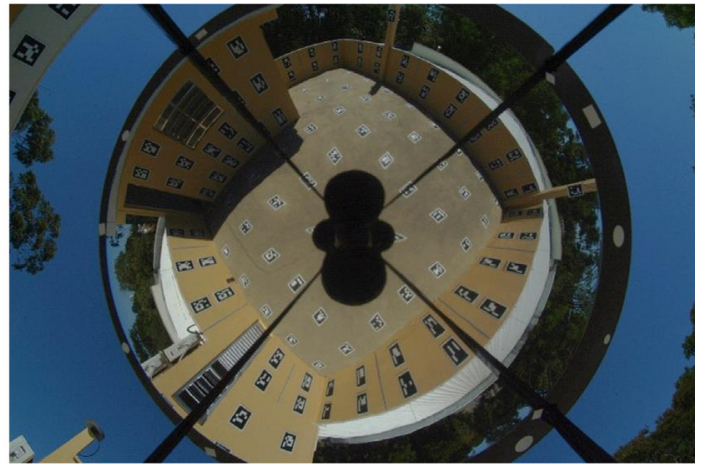

(d)

Fig. 7. Images acquired using the catadioptric omnidirectional system in the field calibration.

$$
\left(\begin{array}{c}
X_{\text {cone }} \\
Y_{\text {cone }} \\
Z_{\text {cone }}
\end{array}\right)=\left(R_{\text {Cone }}^{O b j}\right)^{T}\left(\begin{array}{c}
X-X_{V} \\
Y-Y_{V} \\
Z-Z_{V}
\end{array}\right)
$$

where $R_{\text {Cone }}^{O b j}$ is the rotation matrix corresponding to the conic mirror with respect to the object space reference systems. $X_{V}, Y_{V}$ and $Z_{V}$ are the coordinates of the origin of the conic mirror reference system (conic mirror apex) in the object reference system. Inserting Eq. (13) into Eqs. (10) and (12), the final equations are derived and then linearized and used in the unified approach to the least squares adjustment. A script was implemented in the Matlab software to estimate the exterior orientation parameters by using bundle block adjustment.

In the bundle block adjustment with the four images (Fig. 7), 347 GCPs were used, with a standard deviation of $3 \mathrm{~mm}$, and 901 reflection points, with a standard deviation of $0.2 \mathrm{~mm}$. The standard deviation for the reflection points were established through variance propagation with the equations used in monoplotting. More details about the estimation of the GCPs coordinates are presented in Marcato Junior et al. (2015).

Table 4 presents the results achieved in the indirect orientation process.

The analysis of Table 4 shows that the a posteriori variance factor is approximately 1 and is thus equal to the apriori variance factor. The application of a chi-square test shows that the null hypothesis is accepted, with a significance level of 5\%. In the bundle block adjustment, 15 checkpoints (Fig. 8) were used for the accuracy assessment. Table 5 presents the mean of discrepancies and RMSE in the checkpoint coordinates.

The analysis of Table 5 demonstrates that the mean of discrepancies and RMSE are smaller for the $Z$ coordinate. This is consistent with the results presented in Table 4 , where the estimated standard deviation for $Z_{v}$ is smaller than the standard deviation for the $X_{v}$ and $Y_{v}$ parameters. The object reference system depicted in Fig. 9 justifies this result. The ray intersection generates major uncertainties

Table 4

Parameters and standard deviation relating the conic mirror and object space reference systems estimated with proposed approach.

\begin{tabular}{|c|c|c|c|c|c|c|}
\hline Im. & $\omega_{\text {Cone }}^{\text {Obj }}\left({ }^{\circ}\right)$ & $\phi_{\text {Cone }}^{\text {obj }}\left({ }^{\circ}\right)$ & $\kappa_{\text {Cone }}^{\text {obj }}\left({ }^{\circ}\right)$ & $X_{V}(\mathrm{~m})$ & $Y_{V}(\mathrm{~m})$ & $Z_{V}(\mathrm{~m})$ \\
\hline 1 & $\begin{array}{l}178.69852 \\
(0.00754)\end{array}$ & $\begin{array}{l}0.22962 \\
(0.00668)\end{array}$ & $\begin{array}{l}258.74924 \\
(0.01589)\end{array}$ & $\begin{array}{l}458016.3326 \\
(0.0018)\end{array}$ & $\begin{array}{l}7553601.2968 \\
(0.0017)\end{array}$ & $\begin{array}{l}439.2728 \\
(0.0004)\end{array}$ \\
\hline 2 & $\begin{array}{l}179.26627 \\
(0.00766)\end{array}$ & $\begin{array}{l}1.19849 \\
(0.00698)\end{array}$ & $\begin{array}{l}184.13851 \\
(0.01647)\end{array}$ & $\begin{array}{l}458016.3262 \\
(0.0017)\end{array}$ & $\begin{array}{l}7553601.3396 \\
(0.0017)\end{array}$ & $\begin{array}{l}439.2760 \\
(0.0004)\end{array}$ \\
\hline 3 & $\begin{array}{l}179.86128 \\
(0.00709)\end{array}$ & $\begin{array}{l}1.33497 \\
(0.00646)\end{array}$ & $\begin{array}{l}141.94872 \\
(0.01476)\end{array}$ & $\begin{array}{l}458016.3009 \\
(0.0015)\end{array}$ & $\begin{array}{l}7553601.3338 \\
(0.0014)\end{array}$ & $\begin{array}{l}439.2721 \\
(0.0004)\end{array}$ \\
\hline 4 & $\begin{array}{l}180.23329 \\
(0.01346)\end{array}$ & $\begin{array}{l}-0.50787 \\
(0.01167)\end{array}$ & $\begin{array}{l}80.18984 \\
(0.02338)\end{array}$ & $\begin{array}{l}458016.3654 \\
(0.0030)\end{array}$ & $\begin{array}{l}7553601.2635 \\
(0.0028)\end{array}$ & $\begin{array}{l}440.4581 \\
(0.0008)\end{array}$ \\
\hline \multicolumn{2}{|c|}{$\begin{array}{l}\text { Iterations: } 4 \\
\text { Redundancy: } 2634\end{array}$} & & & \multicolumn{2}{|l|}{$\begin{array}{l}\sigma_{0}=1 \\
\hat{\sigma}_{0}=1.02\end{array}$} & \\
\hline
\end{tabular}



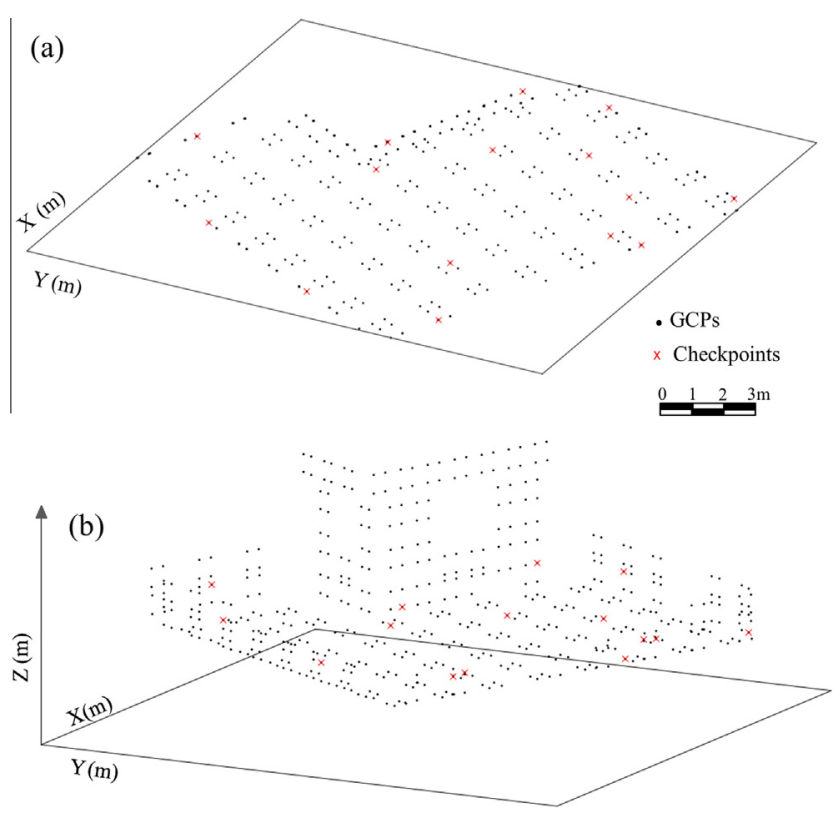

Fig. 8. Spatial distribution of checkpoints.

Table 5

Mean of discrepancies and RMSE in the checkpoint coordinates.

\begin{tabular}{llrl}
\hline & $d X(\mathrm{~m})$ & $d Y(\mathrm{~m})$ & $d Z(\mathrm{~m})$ \\
\hline Mean & 0.008 & -0.007 & 0.001 \\
RMSE & 0.024 & 0.032 & 0.007 \\
Maximum & 0.045 & 0.072 & 0.012 \\
\hline
\end{tabular}

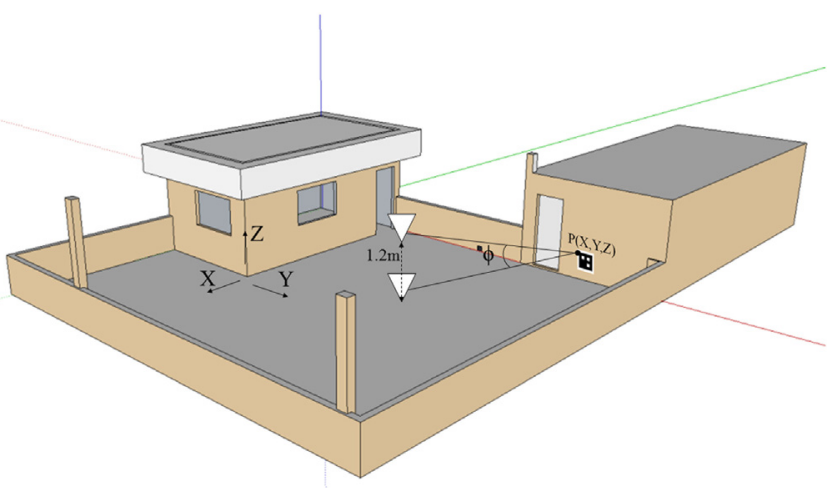

Fig. 9. Object reference system and the geometry for the ray intersection regarding the conic mirror.

in the $X$ and $Y$ coordinates. To minimize these differences, images should be acquired with a larger parallactic angle $(\phi)$.

In the current experiment, only one image (Image 4) was acquired 1.2 meters above the ground (Fig. 9 and Table 4). Fig. 10 presents the residual distributions in the checkpoint coordinates.

The residuals in Fig. 10 present a random behavior. The RMSE for the $X$ and $Y$ coordinates is approximately $3 \mathrm{~cm}$, and for the $Z$ coordinates, it is less than $1 \mathrm{~cm}$. These results can be explained by the mirror surface imperfections, the residuals resulting from the interior calibration process and the image acquisition geometry.
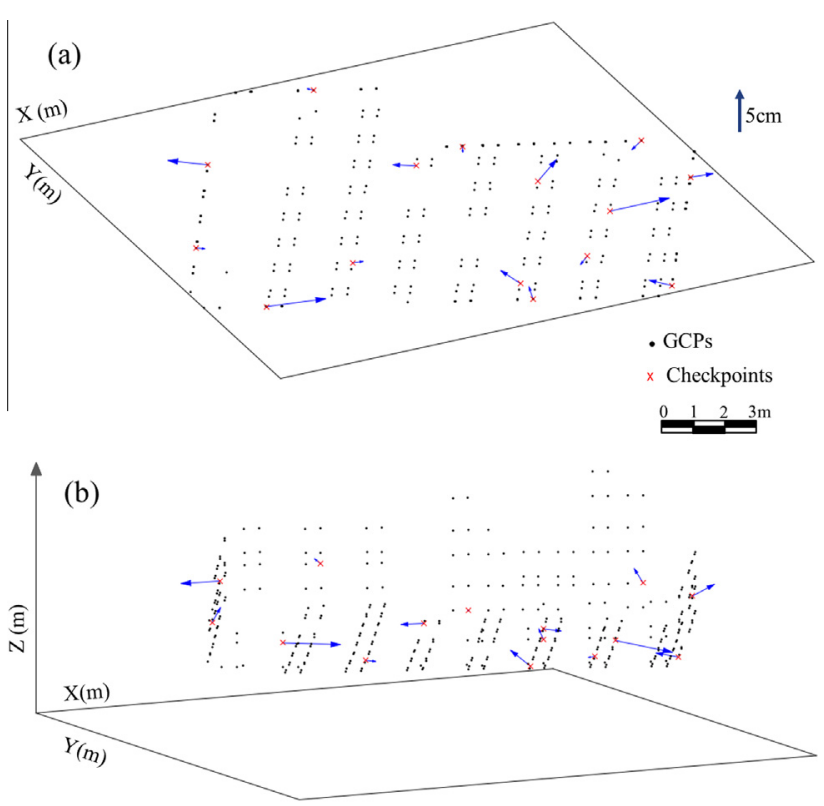

Fig. 10. Residuals (multiplied by 20) distribution in the checkpoint coordinates.

Table 6

Discrepancies in the conic apex $Z_{V}$ coordinate.

\begin{tabular}{llll}
\hline Im. & Estimated $(\mathrm{m})$ & Measured $(\mathrm{m})$ & Discrepancies $(\mathrm{m})$ \\
\hline 1 & 439.2728 & 439.28 & -0.0072 \\
2 & 439.2760 & 439.28 & -0.0040 \\
3 & 439.2721 & 439.28 & -0.0079 \\
Mean & & & -0.0063 \\
RMSE & & & 0.0080 \\
\hline
\end{tabular}

Images 1,2 and 3 were acquired above a target (GCP), the $Z$ coordinate of which is known $(438.753 \mathrm{~m})$. Additionally, the vertical distance between the target and the conic mirror apex $(52.7 \mathrm{~cm} \pm 1 \mathrm{~cm})$ was measured. Then, the approximated $Z$ coordinate of the conic mirror apex was determined to be $439.28 \mathrm{~m}$ for the three images. Table 6 presents the discrepancies between the estimated and measured values.

The RMSE presented in Table 6 is smaller than the precision attained in the vertical distance between the target and the conic mirror apex measurement $( \pm 1 \mathrm{~cm})$. The results presented in Table 6 are consistent with the RMSE of the $Z$ coordinates of the checkpoints shown in Table 5.

The results reached in the experiments validated the technique that was developed for calibrating a catadioptric omnidirectional system that is composed of a wide-angle camera and a conic mirror, which is a catadioptric that does not satisfy the single viewpoint property.

\section{Conclusion}

The calibration techniques for a catadioptric omnidirectional system were generally developed for central catadioptric systems, which follow the single viewpoint property.

Spacek (2003) presented many advantages of omnidirectional catadioptric systems that are composed of a camera and a conic mirror over other omnidirectional systems. A catadioptric system composed of a camera and a conic mirror does not follow this property; consequently, few works were developed in regards to this system calibration. 
The main aim of this paper was to present a novel technique for calibrating a catadioptric omnidirectional vision system that is composed of a conic mirror and a wide-angle lens camera. The developed technique has three steps: camera calibration; conic mirror modeling; and estimating the transformation parameters between the camera and the conic mirror reference systems. The main advantage of the developed technique is that it does not require physical alignment between the conic mirror and the camera axis. Another advantage is the reduction of the parameters' correlation because the calibration steps are conducted in sequential steps. It is important to note that the technique can be applied to both wide-angle lens cameras and perspective cameras.

In the indirect orientation process with bundle adjustment, an accuracy of $1-3 \mathrm{~cm}$ was achieved in the checkpoint coordinates. To improve the results obtained in the $3 \mathrm{D}$ reconstruction, it is suggested to reduce the mirror imperfections through a more sophisticated mirror building and polishing process. After calibration, the developed system can be used in photogrammetric activities that require centimeter level accuracy, including mobile applications.

In future works, mathematical modeling for a 3D intersection using images acquired with the catadioptric omnidirectional system used in the current paper will be developed. Experiments comparing the developed technique with others (Agrawal et al., 2011; Schöenbein et al., 2014) will also be conducted. The integration of the catadioptric system with direct orientation system (GNSS and IMU) will be performed, reducing the need for GCPs. The development of a technique integrating all the calibration steps will also be a subject of study in a future research.

\section{Acknowledgements}

The authors would like to acknowledge the support of FAPESP (Fundação de Amparo à Pesquisa do Estado de São Paulo), through a PhD Scholarship (p. 2010/16439-3), CNPq (Conselho Nacional de Desenvolvimento Científico e Tecnológico) and FUNDECT (Fundação de Apoio ao Desenvolvimento do Ensino, Ciência e Tecnologia do Estado de Mato Grosso do Sul), through research grants (p. 477738/2009-5, p. 305111/2010-8, p. 456149/2014-7 and p. 59/300.066/2015). The authors would also like to acknowledge Dr. André Luís Olivete, who built the omnidirectional vision system.

\section{References}

Abraham, S., Förstner, W., 2005. Fish-eye-stereo calibration and epipolar rectification. ISPRS J. Photogram. Remote Sens. 59 (5), 278-288.

Agrawal, A., Taguchi, Y., Ramalingam, S., 2011. Beyond alhazen's problem: Analytical projection model for non-central catadioptric cameras with quadric mirrors. In: Proc. IEEE Computer Vision and Pattern Recognition (CVPR), Colorado Springs, 20-25 June. pp. 2993-3000.

Baker, S., Nayar, S.K., 1999. A theory of single-viewpoint catadioptric image formation. Int. J. Comput. Vision 35 (2), 175-196.

Barreto, J., Araújo, H., 2002. Geometric properties of central catadioptric line images. In: Proc. 7th European Conference on Computer Vision, Copenhagen, 27 May02 June. pp. 237-251.

Brown, D.C., 1971. Close-range camera calibration. Photogram. Eng. 37 (8), 855 866.

Burbridge, C., Spacek, L., 2006. Omnidirectional vision simulation and robot localisation. In: Proc. TAROS'06, Surrey, 4-6 September. pp. 32-39.

Burbridge, C., Nehmzow, U., Condell, J., 2008. Omnidirectional projections with cone mirror and single mirror stereo. In: Proc. 8th Workshop on Omnidirectional Vision, Camera Networks and Non-classical Cameras, Marseille, 12-18 October. $11 \mathrm{p}$.

Cauchois, C., Brassart, E., Drocourt, C., Vasseur, P., 1999. Calibration of the omnidirectional vision sensor: Syclop. In: Proc. IEEE International Conference on Robotics and Automation, Detroit, 10-15 May. pp. 1287-1292.
Charles, J.R., 2009. Review of Samyang $8 \mathrm{~mm}$ f/3.5 proportional projection ultrawide angle lens. <http://www.versacorp.com/vlink/jcreview/sy8rv9jc.pdf>. (accessed 13.02.15).

Conrady, A., 1919. Decentered lens systems. Mon. Not. R. Astron. Soc. 79, 384-390.

El-Hakim, S.F., 1986. Real-time image meteorology with CCD cameras. Photogram. Eng. Remote Sens. 52 (11), 1757-1766.

Garrido-Jurado, S., Muñoz-Salinas, R., Madrid-Cuevas, F.J., Marín-Jiménez, M.J., 2014. Automatic generation and detection of highly reliable fiducial markers under occlusion. Pattern Recogn. 47 (6), 2280-2292.

Habib, A.F., Morgan, M.F., 2003. Automatic calibration of low cost digital cameras. SPIE J. Optic. Eng. 42 (4), 948-955.

Hughes, C., Denny, P., Jones, E., Glavin, M., 2010. Accuracy of fish-eye lens models. Appl. Opt. 49 (17), 3338-3347.

Joung, I.S., Cho, H.S., 1998. An active omni-directional range sensor for mobile robot navigation. Cont. Eng. Pract. 6 (3), 385-393.

Kang, S.B., Szeliski, R., 1997. 3-D scene data recovery using omnidirectional multibaseline stereo. Int. J. Comput. Vision 25 (2), 167-183.

Lin, S.S., Bajcsy, R., 2001. Single cone mirror omni-directional stereo. Technical Report, code MS-CIS-01-03.

López-Nicolás, G., Sagües, C., 2010. Catadioptric camera model with conic mirror. In: Proc. British Machine Vision Conference, Aberystwyth, 31 August-3 September. pp. 114.1-114.10.

Luber, A., Reulke, R., 2010. A unified calibration approach for generic cameras. Int. Arch. Photogram. Remote Sens. Spatial Inform. Sci. XXXVIII B5, 399-404.

Makarovik, B., 1973. Digital mono-ploters. I.T.C J. 1, 101-122.

Marcato Junior, J., Tommaselli, A.M.G., 2013. Exterior orientation of CBERS-2B imagery using multi-feature control and orbital data. ISPRS J. Photogram. Remote Sens. 79, 219-225.

Marcato Junior, J., Moraes, M.V.A., Tommaselli, A.M.G., 2015. Experimental assessment of techniques for fisheye camera calibration. Boletim de Ciências Geodésicas 21 (3), 637-651.

Mei, C., Rives, P., 2007. Single view point omnidirectional camera calibration from planar grids. In: Proc. IEEE International Conference on Robotics and Automation, Roma, 10-14 April. pp. 3945-3950.

Mikhail, E.M., Ackerman, F., 1976. Observations and Least Squares, first ed., 497 IEP, New York.

Nalwa, V.S., 1996. A true omnidirectional viewer. Bell Laboratories Technical Report, Holmdel, NJ 07733.

Puig, L., Bastanlar, Y., Sturm, P., Guerrero, J.J., Barreto, J., 2011. Calibration of central catadioptric cameras using DLT-like approach. Int. J. Comput. Vision 93 (1), $101-114$.

Puig, L., Bermúdez, J., Sturm, P., Guerrero, J.J., 2012. Calibration of omnidirectional cameras in practice. A comparison of methods. Comput. Vis. Image Underst. 116 (1), 120-137.

Richter, K., Mader, D., Seidl, K., Maas, H.-G., 2013. Development of a geometric model for an all-reflective camera system. ISPRS J. Photogram. Remote Sens. 86, 41-51.

Scaramuzza, D., Martinelli, A., Siegwart, R., 2006. A flexible technique for accurate omnidirectional camera calibration and structure from motion. In: Proc. IEEE International Conference on Computer Vision System, New York, 4-7 January. $8 \mathrm{p}$.

Schneider, D., Schwalbe, E., Maas, H.-G., 2009. Validation of geometric models for fisheye lenses. ISPRS J. Photogram. Remote Sens. 64 (3), 259-266.

Schöenbein, M., Strauss, T., Geiger, A., 2014. Calibrating and centering quasi-central catadioptric cameras. In: Proc. IEEE International Conference on Robotics and Automation, Hong Kong, 31 May-7 June. pp. 4443-4450.

Silva, S.L.A., Tommaselli, A.M.G., Artero, A.O., 2014. Utilização de alvos codificados do tipo ArUco na automação do processo de calibração de câmaras. Boletim de Ciências Geodésicas 20 (3), 626-646 (in Portuguese).

Spacek, L., 2003. Omnidirectional perspective and stereopsis with conical mirrors. Research Report CTU-CMP-2003-12.

Spacek, L., 2005. A catadioptric sensor with multiple viewpoints. Robot. Autonom. Syst. 51 (1), 3-15.

Sturm, P., Ramalingam, S., Tardif, J., Gasparini, S., Barreto, J., 2011. Camera models and fundamental concepts used in geometric computer vision. Comput. Graph. Vis. 6 (1-2), 1-183.

Tan, K., Hua, H., Ahuja, N., 2004. Multiview panoramic cameras using mirror pyramids. PIEE Trans. Pattern Anal. Mach. Intell. 26 (7), 941-946.

Tommaselli, A.M.G., Reiss, M.L.L., 2005. A photogrammetric method for single image orientation and measurement. Photogram. Eng. Remote Sens. 71 (6), 727-732.

Tommaselli, A.M.G., Marcato Junior, J., Moraes, M.V.A., Silva, S.L.A., Artero, A.O., 2014. Calibration of panoramic cameras with coded targets and a 3D calibration field. Int. Arch. Photogram. Remote Sens. Spatial Inform. Sci. XL-3/W1, pp. 137142 .

Yagi, Y., Kawoto, S., Tsuji, S., 1994. Real time omnidirectional image sensor sensor (COPIS) for vision-guided navigation. IEEE Trans. Robot. Automat. 10 (1), 11-22.

Yagi, Y., 1999. Omnidirectional sensing and its applications. IEICE Trans. Inf. \& Sist. E82-D (3), 568-579. 